\title{
Interactive Qualitative Analysis - A Novel Methodology for Qualitative Research
}

\author{
Dr Karen Bargate \\ Senior Lecturer: Managerial Accounting and Financial \\ Management, School of Accounting, Economics and Finance \\ University of KwaZulu-Natal; Durban; South Africa \\ bargate@ukzn.ac.za
}

\author{
Doi:10.5901/mjss.2014.v5n20p11
}

\begin{abstract}
This study investigates the use of Interactive Qualitative Analysis (IQA) (Northcutt \& McCoy, 2004), as a methodology, to develop an understanding of how Managerial Accounting and Financial Management students experienced learning in a writing intensive tutorial programme. IQA is an innovative methodology providing a structured approach to conducting qualitative research. Participants or constituents are actively engaged in data collection and analysis. Using thematic content analysis of the data, they articulate their experiences of the phenomenon and identify emergent themes, or affinities and the relationship between the affinities. The outcome of the IQA process is a Systems Influence Diagram, a visual representation of the phenomenon, constructed through the lens of the constituents. What sets IQA apart from other forms of qualitative inquiry is that it provides an audit trail of transparent and traceable procedures where the constituents, and not the researcher as expert, do the analysis and interpretation of their data. The analysis of the data is as far as possible free from researcher bias as the researcher is merely a facilitator of the process. The key value of this paper is that it offers a practical methodological approach to using IQA in qualitative accounting education research, in particular, and business management education research, in general.
\end{abstract}

Keywords: affinities; constituents; Interactive Qualitative Analysis; qualitative research; Systems Influence Diagram

\section{Introduction}

Qualitative research is an inquiry process aimed at understanding social or human problems based in a natural setting. With qualitative research there are multiple perspectives of reality, which are subjective and open to researcher bias as the researcher actively participates in the research process and analyses the data and in so doing builds an understanding of a complex set of processes while reporting on the views of participants (Creswell 1994, p. 1,2). This process raises issues related to lack of rigour, trustworthiness and reliability (Creswell, 2009; Denzin \& Lincoln, 1998a, 1998b) which beset qualitative research. Interactive Qualitative Analysis (IQA) (Northcutt \& McCoy, 2004) is an innovate approach to qualitative research, which seeks to minimise the power relations and biases traditionally associated with qualitative research (Paz Dennen, 2005; Tabane \& Human-Vogel, 2010). With IQA, participants are actively engaged in collecting and analysing the data. The outcome of the IQA process is a System Influence Diagram (SID), which is "a visual representation of a phenomenon prepared according to rigorous and replicable rules for the purpose of achieving complexity, simplicity, comprehensiveness and interpretability" (Northcutt \& McCoy 2004, p. 41).

The value of this paper lies in offering a practical methodological approach to using IQA in a qualitative accounting education study exploring Managerial Accounting and Financial Management (MAFM) students' experiences of learning in a writing intensive tutorial (WIT) programme. The detailed description of the IQA methodology process presented in this study could be applied to other studies in areas of business management research.

The remainder of this paper is structured as follows: IQA as a qualitative research design method is considered followed by a discussion of the ideology of IQA. Thereafter, the IQA process is described using MAFM students' experiences of learning in a WIT programme as an illustrative example. The final section concludes the paper.

\section{Interactive Qualitative Analysis}

IQA (Northcutt \& McCoy, 2004) is a structured approach to qualitative research design (du Preez \& du Preez, 2012; Mampane \& Bouwer, 2011; Northcutt \& McCoy, 2004). IQA uses focus groups to produce a systematic representation of a phenomenon from participants' experiences of the phenomenon being studied. IQA is based on the premise that those 
closest to the phenomenon being studied, the participants or constituents in IQA terminology, are best suited to construct a graphic representation of the systems' influences and outcomes. In the early stages of analysis, constituents' voices are privileged over that of the researcher. Constituents are defined as "a group of people who have a shared understanding of the phenomenon" (Northcutt \& McCoy 2004, p. 44), and are selected on the basis of their power over and distance from (closeness to) the phenomenon under investigation. With traditional qualitative research, the researcher generates the themes: however, with IQA, the constituents are responsible for the open coding of the data and generating themes or affinities.

IQA as a research design is consistent with the principles of social constructivism because it "privileges the nature of socially constructed meaning" (Northcutt \& McCoy 2004, p. 4). It allows the group to construct categories of meaning, affinities, and the role of the researcher is that of facilitator. The affinities provide the protocol for individual semistructured interviews where constituents' experiences of the phenomenon can be further explored. An Interrelationship Diagram (IRD) is developed portraying the cause and effect, or influence between affinities. A Systems Influence Diagram (SID) is the final outcome. Northcutt and McCoy $(2004$, p.41) state: "The product of an IQA study is a visual representation of a phenomenon prepared according to rigorous and replicable rules for the purpose of achieving complexity, simplicity, comprehensiveness and interpretability."

In the collection and analysis of data, constituents articulate their experiences of the phenomenon and develop affinities in focus groups thus meaning is socially constructed. This process effectively reduces issues of trustworthiness, dependability and conformability (Tabane, 2010). The role of the researcher is that of facilitator of the process, minimising the researcher's power and influence over constituents during data analysis. "The researcher's role then moves from designer to facilitator, teaching the group members the process and guiding them to generate and analyze their own data with minimal external influence." (Northcutt \& McCoy 2004, p. 44). Consequently, the researcher's biases and prejudices are minimised in the process.

\section{The Ideology of IQA}

In this section, the ontological and epistemological assumptions as they inform the research method and design (Cohen, Manion \& Morrison 2007, p. 8 - 20) are described.

\subsection{Ontological perspective}

"IQA presumes that knowledge and power are largely dependent" (Northcutt \& McCoy 2004, p. 16). The constituents are selected because they hold the power and knowledge (which are inextricably linked) of the phenomenon being studied through their membership of a particular group. In this study, constituents were selected due to their membership of the WIT programme which provided them with authority to reflect on their experience of learning in that programme.

"IQA presumes that the observer and the observed are dependent or ... interdependent" (Northcutt \& McCoy 2004, p. 16). IQA challenges the traditional assumptions of qualitative research which suggest that the role of participants is to generate data, which only the researcher is qualified to analyse. With IQA, constituents generate and interpret their own data while the researcher facilitates the process.

"The object of research in IQA is clearly reality in consciousness" (Northcutt \& McCoy 2004, p. 16). The selection of constituents is made from among those closest to the phenomenon, and in this study, students who participated in the WIT programme. Data collection is undertaken in focus groups thus reality is socially constructed by members of the group. Follow-up interviews are used to further probe individual meanings of the constructs. The central construct of this study was learning, and the focus group format provided constituents with the opportunity to chronicle the processes by which they learned MAFM in the WIT programme.

\subsection{Epistemological perspective}

"IQA insists that both deduction and induction are necessary to the investigation of meaning (Northcutt \& McCoy 2004, p. 16). In IQA, categories of meaning or affinities are socially constructed by constituents in focus groups through induction. Affinities are then defined and refined by the constituents (induction and deduction). In the final step, participants deductively explore the relationship between constructs. The IQA process of coding corresponds with the traditional classes of the analysis of coding - emergent, axial and theoretical (Northcutt \& McCoy 2004, p. 16).

"IQA contends that decontextualized descriptions are useful and possible as long as they are backed up or grounded ... by highly contextualized ones" (Northcutt \& McCoy 2004, p. 17). The researcher aids the reader by providing 
the context within which the research was conducted. Northcutt and McCoy (2004) refer to the Denzin and Lincoln (1998a, p. 3) metaphor of the bricoleur as quilt maker. With IQA, the group, create their own interpretive quilt of meaning or bricolage.

"IQA is clearly favorable to theory, both from the point of view of inducing theory and of testing it." (Northcutt \& McCoy 2004, p. 17). The outcome of IQA is a mindmap of a group's or an individual's mental models of a particular phenomenon. The relationship between constructs can then be theorised. The voices of those closest to the phenomenon, constituents on the WIT programme, are privileged over that of the researcher.

\section{The IQA Process}

The first phase of the IQA entails the selection of constituents to participate in the study, focus group interviews, and generation of a composite visual representation of the phenomenon. The second phase of data collection is semistructured interviews to further probe the constituents' experiences of affinities developed in the focus groups and subsequently theorise the relationships. Due to length constraints, this phase of the IQA process is not described in this paper.

\subsection{Identifying constituents}

Constituents are selected according to the criteria of "distance and power" (Northcutt \& McCoy 2004, p. 69) in relation to the phenomenon being studied. Constituents for this study were a purposive sample of 15 MAFM students who voluntarily participated in an 18-week WIT programme. They shared a direct and common experience of the phenomenon, and in focus group sessions actively engaged in generating the data, coding it and eliciting affinities or themes.

\subsection{Focus group sessions}

During the focus group phase of the IQA process, constituents' experiences of the WIT programme were probed using silent brainstorming for data generation. Constituents shared common perceptions of the WIT programme, however, each constituent perceived the experience in different ways.

At the commencement of the focus groups, constituents were asked to relax, make themselves comfortable, close their eyes, take a few deep breaths and clear their minds. They were then asked to silently reflect on their experiences of learning in the WIT programme. In order to initiate reflection, an issue statement was provided by the facilitator. An issue statement is "used to deconstruct and operationalise the research question" (Mampane \& Bouwer 2011, p. 117). After a brief discussion of the issue statement, constituents spent about 10 minutes silently reflecting on their experiences and then wrote their reflections on Post-its. There was no limit to the number of Post-its each constituent could write, however, there must be only one thought, experience or, word, per Post-it. Brainstorming is conducted in silence to eliminate the influence of dominant members of the group. The facilitator is there to provide a safe environment to guide the process and encourage constituents to write without censoring their thoughts, until they have exhausted their ideas (Northcutt \& McCoy 2004, p. 69). Once everyone has finished writing, Post-its are affixed randomly on a wall.

\subsubsection{Affinity analysis}

Once the brainstorming is complete, then the analysis of the data generated commences. In the clarification stage, constituents are asked to begin by silently reading the Post-its which have been affixed to a wall. The facilitator then reads each Post-it aloud to ensure that constituents understand what is written and to clarify if required.

\subsubsection{Inductive coding}

In the clustering stage, constituents arranged the Post-its in sets with common meanings. This continues until consensus is reached with the placement of Post-its into affinity groupings yielding a collective view. Again this process should be conducted silently to prevent dominant individuals or the facilitator monopolizing the process. 


\subsubsection{Axial coding}

Constituents then reviewed the Post-its under each grouping to ensure that they were under the correct themes. A Post-it was placed at the top of each thematic grouping naming it. A limited amount of refining of Post-its was required to ensure that all of the Post-its were correctly categorised into the relevant affinity. Affinities produced by each group were aggregated and nine common affinities emerged. The nine affinities identified were: challenging; critique; enjoyable; interactive; personal confidence, positive structure; study technique and test preparation; understanding; and written tasks.

The IQA focus group process takes between three and four hours to complete (Lin \& Tu, n.d.), which can be a disadvantage of the IQA process if constituents do not have that length of time available (LodewyckX, 2005). Once affinities have been identified, a detailed affinity relationship table is constructed.

\subsection{Detailed Affinity Relationship Table}

An Affinity Relationship Table (ART) is completed by constituents to record an analysis of each pair of affinity relationships. The relationship can be one of three,

- Understanding $\rightarrow$ interaction (understanding influences interaction)

- Understanding $\leftarrow$ interaction (interaction influences understanding)

- Understanding < > interaction (no relationship between the affinities)

Further, constituents are asked to write a hypothesis "that reflects their experiences and that supports the cause and effect relationship" (Northcutt \& McCoy 2004, p. 152) between affinity pairs. This could be in the form of a short "ifthen' statement. The responses are taken at face value as the true meaning of how constituents conceptualised the affinity relationships (Human-Vogel 2006, p. 619).

The ART can be completed individually or in dyads or triads. In this study, seven constituents chose to complete it individually and eight chose to do it with a friend, resulting in four dyads. The dyads took much longer to complete than the individuals as they had to reach consensus on the direction of the arrows. The majority of the constituents took far longer to complete the exercise than the facilitator had anticipated - more than 45 minutes. It is suggested that a sufficient block of time is set aside to complete the exercise.

With IQA it is possible to analyse the ART at either a group composite level or an individual level to create the IRD. In this study, relationships were analysed on a group level to determine the group's composite understanding of the phenomenon (Human-Vogel \& Mahlangu, 2009). Pareto protocol is a statistical method that was used to determine the optimal number of relationships to comprise the IRD. It is based on the fundamental principle that "A minority of the relationships in any system will account for a majority of the variation within the system." (Northcutt \& McCoy 2004, p. 157). This optimum number of relationships will be at the point where power reaches a maximum. Using individual and dyad ARTs, each relationship frequency is tallied, entered on a spreadsheet and the total number of votes for each relationship calculated. A total of 344 votes were cast for all combinations of affinity pairs. The outcome of the process is the frequency of each relationship in affinity pair order - see Annexure I. An appraisal of Annexure I indicates that comparatively few of the possible 72 relationships account for most of the variance, consistent with the Pareto principle. Figure 1 is an illustration of the variance accounted for by each succeeding relationship.

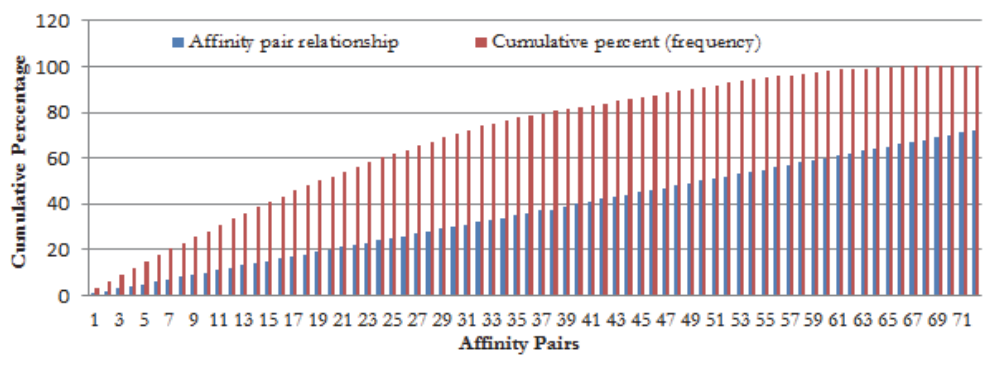

Figure 1: Cumulative percent - total relationships

As shown in Figure 2, power reaches a maximum (29.683) at 32 relationships which accounts for $74.128 \%$ of the 
variation in this system (Annexure I). Accordingly the first 32 affinity relationships will be included in the group IRD.

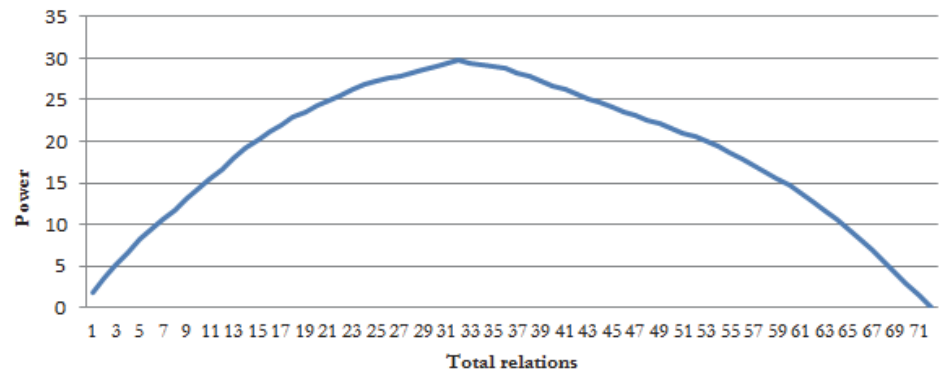

Figure 2: Cumulative percentage - total relationships

When deciding which relationships to include or exclude from the composite group IRD, the last two columns of the Pareto table are pivotal in determining where to set the cut-off point as relationships are displayed in decreasing order of frequency. Relationships 67 to 72 (Annexure I) are excluded as they failed to attract a single vote. However the decision still needs to be made whether, in the composite IRD, to account for relationships such as those between 55 and 66 which attracted one or two votes. The elegance of the IQA is based on the trade-off between accounting "for maximum variation in the system ... while minimizing the number of relationships in the interest of parsimony" (Northcutt \& McCoy 2004, p. 160).

\subsection{Creating a group composite: the Inter Relationship Diagram}

The IRD is created from the first 32 affinity pair relationships (Annexure I), and this is the first step in rationalising the system (Northcutt \& McCoy 2004, p. 170). Each affinity relationship is mapped to create the composite IRD. The direction of the arrow in each affinity pairing determines which affinity is influenced by which. Up arrows represent the row driving the column and left arrows represent the column driving the row. For example, the first affinity pairing, $2 \rightarrow 6$ signifies that affinity 2 (understanding), cause, is influencing affinity 6 (enjoyment), effect. The IRD works on a similar principle to double-entry booking as every affinity will have 'two entries' in the tabular IRD and the 'outs' and 'ins' need to balance with the number of affinity relationship pairs (32). Thus affinity 2 will have $\uparrow$ (out) into affinity 6 and the balancing entry will be $\leftarrow$ (in) to affinity 2 from affinity 6 (Table 1 ). Delta $(\Delta)$ is the difference between the 'outs' and 'ins' for each affinity.

Table 1: Composite focus group IRD - sorted in descending order

\begin{tabular}{|c|c|c|c|c|c|c|c|c|c|c|c|c|}
\hline$*$ & $\mathbf{1}$ & $\mathbf{2}$ & $\mathbf{3}$ & $\mathbf{4}$ & $\mathbf{5}$ & $\mathbf{6}$ & $\mathbf{7}$ & $\mathbf{8}$ & $\mathbf{9}$ & out $\uparrow$ & in & $\Delta$ \\
\hline $\mathbf{1}$ & & $\uparrow$ & $\uparrow$ & $\uparrow$ & $\uparrow$ & $\uparrow$ & $\uparrow$ & $\uparrow$ & $\leftarrow$ & 7 & 1 & $\mathbf{6}$ \\
\hline $\mathbf{2}$ & $\leftarrow$ & & $\uparrow$ & $\leftarrow$ & $\uparrow$ & $\uparrow$ & $\uparrow$ & $\uparrow$ & $\uparrow$ & 6 & 2 & 4 \\
\hline $\mathbf{4}$ & $\leftarrow$ & $\uparrow$ & $\leftarrow$ & & $\uparrow$ & $\uparrow$ & $\uparrow$ & $\uparrow$ & $\uparrow$ & 6 & 2 & 4 \\
\hline 3 & $\leftarrow$ & $\leftarrow$ & & $\uparrow$ & $\uparrow$ & $\uparrow$ & $\uparrow$ & & & 4 & 2 & $\mathbf{2}$ \\
\hline $\mathbf{9}$ & $\uparrow$ & $\leftarrow$ & & $\leftarrow$ & $\uparrow$ & & & $\leftarrow$ & & 2 & 3 & -1 \\
\hline $\mathbf{5}$ & $\leftarrow$ & $\leftarrow$ & $\leftarrow$ & $\leftarrow$ & & $\uparrow$ & $\uparrow$ & $\uparrow$ & $\leftarrow$ & 3 & 5 & -2 \\
\hline $\mathbf{6}$ & $\leftarrow$ & $\leftarrow$ & $\leftarrow$ & $\leftarrow$ & $\leftarrow$ & & $\uparrow$ & $\uparrow$ & & 2 & 5 & -3 \\
\hline 7 & $\leftarrow$ & $\leftarrow$ & $\leftarrow$ & $\leftarrow$ & $\leftarrow$ & $\leftarrow$ & & $\uparrow$ & & 1 & 6 & -5 \\
\hline 8 & $\leftarrow$ & $\leftarrow$ & & $\leftarrow$ & $\leftarrow$ & $\leftarrow$ & $\leftarrow$ & & $\uparrow$ & 1 & 6 & -5 \\
\hline
\end{tabular}

*Affinities: 1 - positive structure; 2 - understanding; 3 - written tasks; 4 - challenging; 5 - study technique and test preparation; 6 - enjoyment; 7 - personal confidence; 8 - interaction; 9 - critique

The IRD provided the data needed to determine which affinities were drivers or outcomes. Affinities with positive deltas $(1,2,4,3)$ are drivers or causes and affinities with negative deltas $(9,5,6,7,8)$ are outcomes or effects. Positive structure (1) is the primary driver of the system and personal confidence (7) and interaction (8) are primary outcomes. This classification is indicative of a strong cause or effect relationship between relevant affinities. Understanding (2), challenging (4) and written tasks (3) are secondary drivers and critique (9), study technique and test preparation (5) and 
enjoyment (6) are secondary outcomes.

\subsection{Focus group Systems Influence Diagram (SID)}

\subsubsection{Cluttered SID}

The aggregated focus group data is utilised in drawing the SID. The SID is a visual representation of all relationships of the entire system (Northcutt \& McCoy 2004, p. 174). When drawing the SID primary drivers are placed on the extreme left-hand side and primary outcomes on the extreme right-hand side. Secondary outcomes and drivers are placed between primary drivers and outcomes with drivers placed on the left and outcomes on the right. For every relationship in the IRD (Table 1) an arrow is drawn between the two affinities indicating the direction of cause and effect the product of which is the cluttered SID, see Figure 3. The cluttered SID has limited explanatory value as it is too complex for meaningful analysis. For the SID to have relevance it is a precondition that it be uncluttered by removing redundant links.

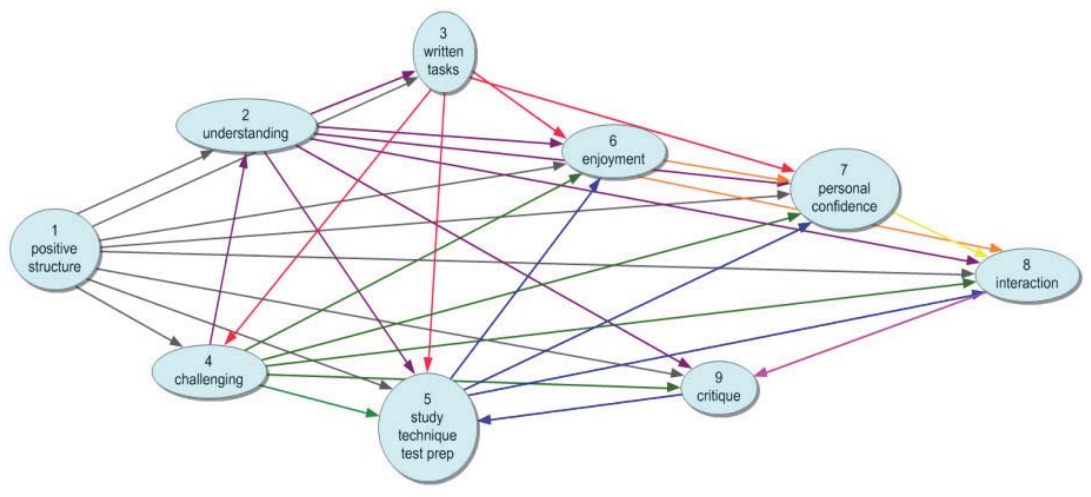

Figure 3: Cluttered SID

\subsubsection{Uncluttered SID}

Once the relationships are examined all redundant links are removed to simplify the diagram, resulting in an uncluttered SID. In the cluttered SID (Figure 3), $1 \rightarrow 3$ would be considered a redundant link because $1 \rightarrow 2$ and $2 \rightarrow 3$, hence the link $1 \rightarrow 3$ can be removed making the system simpler and one that has optimum explanatory power (Northcutt \& McCoy, 2004, p. 177). The process of removing redundant links continued until all had been removed and the resultant uncluttered SID represents MAFM students' experiences of learning in a WIT programme. Figure 4 shows the uncluttered SID.

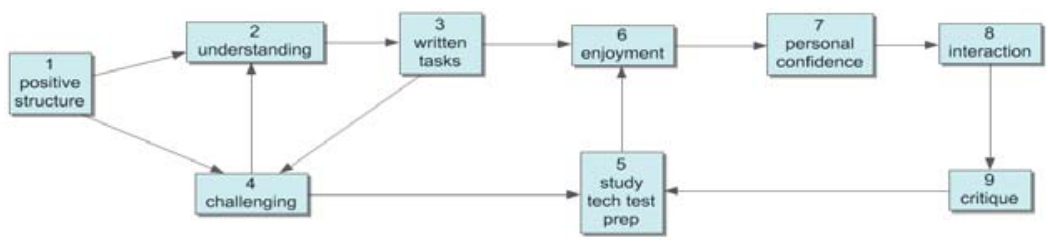

Figure 4: Uncluttered SID

\subsection{A tour through the system}

Looking at the uncluttered SID represented in Figure 4, the following interpretation can be offered. Learning experiences of MAFM students in a WIT programme are driven by the structure of the programme and this influences students' understanding and the challenging nature of the tasks completed. Improved understanding influences the written tasks, 
which in turn influences the challenging nature of the programme and this influences understanding of MAFM. These three elements form a feedback loop. Written tasks influence enjoyment and the challenging nature influences study technique and test preparation. The enjoyment affinity influences personal confidence which influences interaction and this influences critique, which influences studying technique and test preparation. Another feedback loop is formed between affinities $6,7,8,9$ and 5 . The outcome of the system is representative of the interactive nature of the WIT programme.

\subsection{Feedback loops and zooming}

Within the system two feedback loops were identified:

- understanding, written tasks challenging, enjoyment; and

- personal confidence, interaction, critique and study technique and test preparation.

Northcutt and McCoy $(2004$, p. 335) state that a feedback loop consists of at "least three affinities, each influencing the other directly or indirectly". Feedback loops can be renamed by reviewing the components of each subsystem. The process is referred to as "zooming" (Northcutt \& McCoy 2004, p. 335). The substitute name for subsystems is generated by reviewing the axial coding and descriptions together with the placements of the feedback loops within the overall system. The defined components of the first subsystem $(2,3,4)$ suggest intellectually stimulating. The defined components of the second subsystem $(6,7,8,9,5)$ suggest learning is fun. These new "superaffinities" replace the feedback loops via substitution in a new view that is zoomed out (Northcutt \& McCoy 2004, p. 335) (see Figure 5).

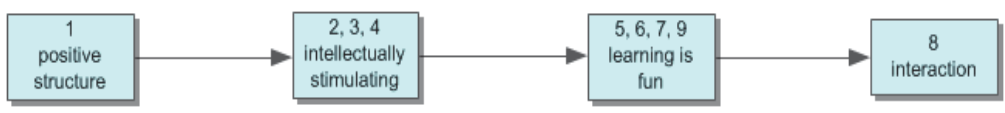

Figure 5: WIT learning experience telephoto view SID

The new system is identical to the first (Figure 4) except that the seven affinities from the two feedback loops have been collapsed or zoomed out into more general terms - 'intellectually stimulating' and 'learning is fun'. This telephoto view SID cannot be zoomed any further and the result is an elegant linear system with no branching.

\section{Conclusion}

This paper discusses IQA as a research method, with application to a WIT programme. In this study, affinities were identified by constituents and presented in the ART from which relationships between affinities were described in the IRD. The culmination of the process (for this study) is the SID which is a visual representation of the system. So far as the researcher has determined, this is the first time IQA has been used in accounting education research.

The value in using IQA as a research design is that it scales back the power relations between researcher and participants. IQA ensures that the voice of the participants is valued and supplements the voice of the researcher. IQA is a complex procedure and if the entire process had been adhered to data collection would have extended beyond the duration of the WIT programme. IQA also imposes time demands on participant subjects. However, using the principles of IQA, the context determines how far the theory can be applied.

\section{References}

Cohen. L., Manion L. \& Morrison K. (2007). Research methods in education. Routledge: London Creswell, J. W. (1994). Research design qualitative and quantitative approaches. Thousand Oaks: SAGE. Creswell, J. W. (2009). Research design qualitative, quantitative and mixed methods approaches. Thousand Oaks: SAGE. Denzin, N. K. \& Lincoln, Y. S. (eds.) (1998a). Collecting and interpreting qualitative materials. Thousand Oaks: SAGE. Denzin, N. K. and Lincoln, Y. S. (eds.) (1998b). The landscape of qualitative research theories and issues. Thousand Oaks: SAGE. du Preez, H. \& du Preez, C. (2012). Taxation students' perceptions of open-book assessment prior to the qualifying examination of South African Chartered Accountants. South African Journal of Accounting Research, 26(1), 119-142.

Human-Vogel, S. (2006). Students' mental modes of positive mood and self-regulation in learning. Psychological Society of South Africa, 36(3), 613-633.

Human-Vogel, S. \& Mahlangu, P. P. (2009). Commitment in academic contexts: first year education students' beliefs about the aspects of self, the lecturer and instruction. South African Journal of Higher Education, 23(2), 309-328. 
Lin, C.-Y. \& Tu, C.-C. (n.d.). Combined IQA approach with a case study for information system requirements. [Online] Available: http://140.130.135.23:8080/dspace/bitstream/TTCIR/405/1/PICMET2010-\%E6\%9E\%97\%E7\%B4\%94\%E9\%9B\%85.pdf.

Lodewyckx, D. P. G. (2005). An Interactive Qualitative Analysis of educational psychology students' self-efficacy beliefs in career counselling. [Online] Available: http://upetd.up.ac.za/thesis/available/etd-12202006-132015/unrestricted/00dissertation.pdf.

Mampane, R. \& Bouwer, C. (2011). The influence of township schools on the resillience of their learners. South African Journal of Education, 31(1), 114-126.

Northcutt, N. \& McCoy, D. (2004). Interactive Qualitative Analysis: a systems method for qualitative research. London: SAGE.

Paz Dennen, V. (2005). Book review: Interactive Qualitative Analysis: a systems method for qualitiative analysis, N. Northcutt, D. McCoy, SAGE (2004). Evaluation and Program Planning, 28, 315-316.

Tabane, R. J. (2010). Integration and learners' feelings of belonging in a desegregated former House of Delegates school. [Online] Available Doctor of Philosophy. University of Pretoria, http://upetd.up.ac.za/thesis/available/etd-05012010-163911/.

Tabane, R. J. \& Human-Vogel, S. (2010). Sense of belonging and social cohension in a desegregated former House of Delegates school. South African Journal of Education, 30(3), 495-504.

Annexure I: Affinities in descending order of frequency with Pareto protocol and power

\begin{tabular}{|c|c|c|c|c|c|c|}
\hline No & $\begin{array}{l}\text { Affinity pair } \\
\text { relationship }\end{array}$ & $\begin{array}{l}\text { Frequency sorted } \\
\text { (descending) }\end{array}$ & $\begin{array}{l}\text { Cumulative } \\
\text { frequency }\end{array}$ & $\begin{array}{l}\text { Cumulative percent } \\
\text { (relation) }\end{array}$ & $\begin{array}{c}\text { Cumulative percent } \\
\text { (frequency) }\end{array}$ & Power \\
\hline 1 & $2 \rightarrow 6$ & 11 & 11 & 1.389 & \begin{tabular}{|c|}
3.198 \\
\end{tabular} & 1.809 \\
\hline 2 & $1 \rightarrow 8$ & 11 & 22 & 2.778 & 6.395 & 3.618 \\
\hline 3 & $2 \rightarrow 7$ & 10 & 32 & 4.167 & 9.302 & 5.136 \\
\hline 4 & $2 \rightarrow 3$ & 10 & 42 & 5.556 & 12.209 & 6.654 \\
\hline 5 & $1 \rightarrow 2$ & 10 & 52 & 6.944 & 15.116 & 8.172 \\
\hline 6 & $4 \rightarrow 8$ & 9 & 61 & 8.333 & 17.733 & 9.399 \\
\hline 7 & $4 \rightarrow 5$ & 9 & 70 & 9.722 & 20.349 & 10.627 \\
\hline 8 & $3 \rightarrow 7$ & 9 & 79 & 11.111 & 22.965 & 11.854 \\
\hline 9 & $3 \rightarrow 6$ & 9 & 88 & 12.500 & 25.581 & 13.081 \\
\hline 10 & $2 \leftarrow 4$ & 9 & 97 & 13.889 & 28.198 & 14.309 \\
\hline 11 & $1 \rightarrow 6$ & 9 & 106 & 15.278 & 30.814 & 15.536 \\
\hline 12 & $1 \rightarrow 5$ & 9 & 115 & 16.667 & 33.430 & 16.764 \\
\hline 13 & $1 \rightarrow 4$ & 9 & 124 & 18.056 & 36.047 & 17.991 \\
\hline 14 & $1 \rightarrow 3$ & 9 & 133 & 19.444 & 38.663 & 19.218 \\
\hline 15 & $7 \rightarrow 8$ & 8 & 141 & 20.833 & 40.988 & 20.155 \\
\hline 16 & $4 \rightarrow 7$ & 8 & 149 & 22.222 & 43.314 & 21.092 \\
\hline 17 & $3 \rightarrow 5$ & 8 & 157 & 23.611 & 45.640 & 22.028 \\
\hline 18 & $3 \rightarrow 4$ & 8 & 165 & 25.000 & 47.965 & 22.965 \\
\hline 19 & $6 \rightarrow 8$ & 7 & 172 & 26.389 & 50.000 & 23.611 \\
\hline 20 & $4 \rightarrow 9$ & 7 & 179 & 27.778 & 52.035 & 24.257 \\
\hline 21 & $4 \rightarrow 6$ & 7 & 186 & 29.167 & 54.070 & 24.903 \\
\hline 22 & $2 \rightarrow 9$ & 7 & 193 & 30.556 & 56.105 & 25.549 \\
\hline 23 & $2 \rightarrow 8$ & 7 & 200 & 31.944 & 58.140 & 26.195 \\
\hline 24 & $1 \rightarrow 7$ & 7 & 207 & 33.333 & 60.174 & 26.841 \\
\hline 25 & $8 \rightarrow 9$ & 6 & 213 & 34.722 & 61.919 & 27.196 \\
\hline 26 & $6 \rightarrow 7$ & 6 & 219 & 36.111 & 63.663 & 27.552 \\
\hline 27 & $5 \leftarrow 9$ & 6 & 225 & 37.500 & 65.407 & 27.907 \\
\hline 28 & $5 \rightarrow 8$ & 6 & 231 & 38.889 & 67.151 & 28.262 \\
\hline 29 & $5 \rightarrow 7$ & 6 & 237 & 40.278 & 68.895 & 28.618 \\
\hline 30 & $5 \rightarrow 6$ & 6 & 243 & 41.667 & 70.640 & 28.973 \\
\hline 31 & $1 \leftarrow 9$ & 6 & 249 & 43.056 & 72.384 & 29.328 \\
\hline 32 & $2 \rightarrow 5$ & 6 & 255 & 44.444 & 74.128 & 29.683 \\
\hline 33 & $2 \leftarrow 5$ & 4 & 259 & 45.833 & 75.291 & 29.457 \\
\hline 34 & $6 \leftarrow 8$ & 4 & 263 & 47.222 & 76.453 & 29.231 \\
\hline 35 & $3 \rightarrow 9$ & 4 & 267 & 48.611 & 77.616 & 29.005 \\
\hline 36 & $7 \leftarrow 9$ & 4 & 271 & 50.000 & 78.779 & 28.779 \\
\hline 37 & $7 \leftarrow 8$ & 3 & 274 & 51.389 & 79.651 & 28.262 \\
\hline 38 & $7 \rightarrow 9$ & 3 & 277 & 52.778 & 80.523 & 27.745 \\
\hline 39 & $6 \leftarrow 9$ & 3 & 280 & 54.167 & 81.395 & 27.229 \\
\hline 40 & $5 \leftarrow 8$ & 3 & 283 & 55.556 & 82.267 & 26.712 \\
\hline
\end{tabular}




\begin{tabular}{|c|c|c|c|c|c|c|}
\hline 41 & $5 \leftarrow 7$ & 3 & 286 & 56.944 & 83.140 & 26.195 \\
\hline 42 & $5 \leftarrow 6$ & 3 & 289 & 58.333 & 84.012 & 25.678 \\
\hline 43 & $5 \rightarrow 9$ & 3 & 292 & 59.722 & 84.884 & 25.161 \\
\hline 44 & $4 \leftarrow 9$ & 3 & 295 & 61.111 & 85.756 & 24.645 \\
\hline 45 & $4 \leftarrow 5$ & 3 & 298 & 62.500 & 86.628 & 24.128 \\
\hline 46 & $3 \leftarrow 9$ & 3 & 301 & 63.889 & 87.500 & 23.611 \\
\hline 47 & $3 \leftarrow 7$ & 3 & 304 & 65.278 & 88.372 & 23.094 \\
\hline 48 & $3 \leftarrow 5$ & 3 & 307 & 66.667 & 89.244 & 22.578 \\
\hline 49 & $3 \leftarrow 8$ & 3 & 310 & 68.056 & 90.116 & 22.061 \\
\hline 50 & $2 \leftarrow 8$ & 3 & 313 & 69.444 & 90.988 & 21.544 \\
\hline 51 & $2 \rightarrow 4$ & 3 & 316 & 70.833 & 91.860 & 21.027 \\
\hline 52 & $1 \leftarrow 5$ & 3 & 319 & 72.222 & 92.733 & 20.510 \\
\hline 53 & $1 \rightarrow 9$ & 3 & 322 & 73.611 & 93.605 & 19.994 \\
\hline 54 & $8 \leftarrow 9$ & 3 & 325 & 75.000 & 94.477 & 19.477 \\
\hline 55 & $6 \leftarrow 7$ & 2 & 327 & 76.389 & 95.058 & 18.669 \\
\hline 56 & $4 \leftarrow 6$ & 2 & 329 & 77.778 & 95.640 & 17.862 \\
\hline 57 & $3 \leftarrow 4$ & 2 & 331 & 79.167 & 96.221 & 17.054 \\
\hline 58 & $2 \rightarrow 3$ & 2 & 333 & 80.556 & 96.802 & 16.247 \\
\hline 59 & $1 \rightarrow 2$ & 2 & 335 & 81.944 & 97.384 & 15.439 \\
\hline 60 & $2 \leftarrow 7$ & 2 & 337 & 83.333 & 97.965 & 14.632 \\
\hline 61 & $6 \rightarrow 9$ & 2 & 339 & 84.722 & 98.547 & 13.824 \\
\hline 62 & $4 \leftarrow 8$ & 1 & 340 & 86.111 & 98.837 & 12.726 \\
\hline 63 & $3 \leftarrow 8$ & 1 & 341 & 87.500 & 99.128 & 11.628 \\
\hline 64 & $2 \leftarrow 9$ & 1 & 342 & 88.889 & 99.419 & 10.530 \\
\hline 65 & $1 \leftarrow 7$ & 1 & 343 & 90.278 & 99.709 & 9.432 \\
\hline 66 & $1 \leftarrow 4$ & 1 & 344 & 91.667 & 100.000 & 8.333 \\
\hline 67 & $4 \leftarrow 7$ & 0 & 344 & 93.056 & 100.000 & 6.944 \\
\hline 68 & $3 \leftarrow 6$ & 0 & 344 & 94.444 & 100.000 & 5.556 \\
\hline 69 & $2 \leftarrow 6$ & 0 & 344 & 95.833 & 100.000 & 4.167 \\
\hline 70 & $1 \leftarrow 8$ & 0 & 344 & 97.222 & 100.000 & 2.778 \\
\hline 71 & $1 \leftarrow 6$ & 0 & & 98.611 & 100.000 & 1.389 \\
\hline 72 & $1 \leftarrow 3$ & 0 & 100.000 & 100.000 & 0.000 \\
\hline
\end{tabular}

